Case Report

\title{
A Case Series of Temozolomide in the Management of Refractory Prolactinomas
}

Divya B. Mella ${ }^{1,}{ }^{*}$, Josie Sewell ${ }^{2}$, Edward Pan ${ }^{1}$

1. Department of Neurology and Neurotherapeutics, UT Southwestern Medical Center; E-Mails: dbmella@outlook.com; Edward.Pan@UTSouthwestern.edu

2. Simmons Comprehensive Cancer Center, UT Southwestern Medical Center; E-Mail: Josie.Sewell@UTSouthwestern.edu

* Correspondence: Divya B. Mella; E-Mail: dbmella@outlook.com

Academic Editor: Antonio Meola

Special Issue: Tumors of the Central Nervous System

OBM Neurobiology Received: October 12, 2018

2018, volume 2, issue 4

Accepted: October 30, 2018

doi:10.21926/obm.neurobiol.1804014

Published: October 31, 2018

\begin{abstract}
:
Objective: To report three cases of refractory prolactinomas treated with Temozolomide (TMZ). Background: Prolactinomas account for $40 \%$ of pituitary adenomas. Dopamine agonists (DA) are the first line of treatment followed by surgical resection and radiation. TMZ is an oral chemotherapeutic agent used in gliomas, which has been given to patients with prolactinomas refractory to conventional treatments.

Methods: Retrospective chart review was conducted for refractory prolactinoma patients treated between 2008 and 2018 at UT Southwestern Medical Center (UTSW). Three patients with refractory prolactinomas received oral TMZ at UTSW.

Results: All three patients demonstrated improvement in symptoms upon TMZ treatment, markedly decreased serum prolactin levels (SPRL), as well as radiographic decrease in tumor size.
\end{abstract}

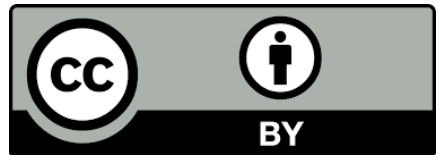

(C) 2018 by the author. This is an open access article distributed under the conditions of the Creative Commons by Attribution License, which permits unrestricted use, distribution, and reproduction in any medium or format, provided the original work is correctly cited. 
Conclusion: TMZ is well tolerated and is a potentially effective treatment for refractory prolactinomas.

\section{Keywords}

Refractory prolactinomas; temozolomide; pituitary adenomas; stereotactic radiosurgery; serum prolactin level

\section{Introduction}

Prolactinomas comprise $40 \%$ of pituitary adenomas [1]. They cause headaches, visual field defects, hypogonadism, galactorrhea and infertility. Most prolactinomas respond to Dopamine agonists (DA) [2]. Surgery is indicated for patients who failed DA treatment or are intolerant to DA. Prolactinomas unresponsive to DA or surgery may need radiosurgery.[3]. Despite multimodal treatments, a subset of prolactinomas progress with rapid growth and invasion into surrounding tissue. TMZ is an oral alkylator used in malignant gliomas which readily crosses the blood brain barrier. Nausea, vomiting, constipation, thrombocytopenia and leucopenia are side effects. Successful management of prolactinomas with TMZ was first reported in 2006 [4, 5]. Approximately $50 \%$ of prolactinomas respond to treatment with $\mathrm{TMZ}$.[6]. A case series evaluating use of $\mathrm{TMZ}$ in refractory prolactinomas reported a response in 4 out of 9 patients. [7]. A recently published large study of 166 patients with aggressive pituitary tumors treated with $\mathrm{TMZ}$ of which majority were corticotroph tumors confirmed that $\mathrm{TMZ}$ is an effective first line treatment of aggressive pituitary tumors and carcinomas. In this retrospective review, patients received TMZ for multiple subtypes of pituitary tumors. Patients with functioning tumors and those who received $\mathrm{TMZ}$ concurrently with radiation therapy had a better response rate [8]. This study is our institutional experience in management of refractory prolactinomas.

\section{Methods}

Retrospective chart reviews were conducted as part of an Institutional Board review exempt study and three refractory prolactinoma patients treated between 2008 and 2018 at UTSW were identified. All patients had at least 3 relapses prior to starting $\mathrm{TMZ}$ treatment. They consented to publication of their clinical data. TMZ was dosed at 150 to $200 \mathrm{mg} / \mathrm{m}^{2} /$ day for five days during each 28-day cycle. All patients had monthly serum prolactin level (SPRL) and bimonthly gadolinium enhanced brain magnetic resonance imaging (MRI) scans.

\section{Patient: 1:}

A 40-year-old female diagnosed with a prolactinoma in 1996 failed treatment with DA, surgery, Cyber knife ${ }^{\circledR}$ and Gamma Knife ${ }^{\circledR}$ radiosurgery. In May 2013, she developed diplopia. Brain MRI showed enlargement of the prolactinoma measuring $3.1 \times 3.4 \times 4.0 \mathrm{~cm}$ (anteroposterior by transverse by craniocaudal (APxTRxCC) with SPRL of $3044.8 \mathrm{ng} / \mathrm{ml}$ (normal range in non-pregnant females: 5-40 
$\mathrm{ng} / \mathrm{ml}$ or $106-850 \mathrm{mIU} / \mathrm{L})$. On completion of 18 cycles of monthly TMZ, her tumor decreased to 2.3 $\mathrm{cm} \times 1.7 \mathrm{~cm} \times 1.8 \mathrm{~cm}$ APXTRxCC. She was monitored with SPRL and brain MRIs every 2 months off TMZ. TMZ was restarted in October 2016 for progression (Figure $1 \mathrm{C}$ ). She completed 21 cycles of TMZ by February 2018 and has stable disease with a SPRL of $8.3 \mathrm{ng} / \mathrm{ml}$. (Figure 2).

Patient 2:

A 70-year-old female was diagnosed with a refractory prolactinoma in 2008. She had failed previous treatment with Dopamine agonists, stereotactic radiosurgery and surgical resection. In June 2014, her SPRL was $3591 \mathrm{ng} / \mathrm{ml}$. (normal range in non-pregnant females: $5-40 \mathrm{ng} / \mathrm{ml}$ or 106-850 $\mathrm{mIU} / \mathrm{L}$ ). She completed 14 cycles of TMZ treatment in August 2015. Her tumor has remained stable off TMZ with normal SPRL in October 2017.

Patient 3:

A 76-year-old male underwent resection and Cyber Knife ${ }^{\circledR}$ radiosurgery of a prolactinoma in October 2009. He was not given DA due to a history of paranoid schizophrenia. He completed 9 cycles of TMZ at a reduced dose of $100 \mathrm{mg} / \mathrm{m}^{2} /$ day due to pancytopenia. His tumor has remained stable off TMZ with normal SPRL in July 2017.

\section{Discussion}

Our series confirmed the reports of previous patients with refractory prolactinomas having good responses and acceptable side effects from TMZ. Optimum dosage of TMZ and duration of treatment (number of cycles) is not known. In our institution, dosage of TMZ was based on the treatment regimen for glioblastoma also known as the "Stupp Protocol" [9]. Patients with refractory prolactinomas in our institution underwent bimonthly gadolinium enhanced brain and pituitary MRI and SPRL measurements every month. In patient 1, after successful completion of TMZ treatment for 12 months, the patient opted to continue TMZ treatment for an additional 6 months given her excellent clinical and radiological response. At recurrence while off TMZ therapy, the patient opted to receive a total of 24 cycles of TMZ treatment and had completed 21 cycles at the time of preparation of this report. Patient 2 opted to discontinue TMZ treatment after 14 cycles due to fatigue. Patient 3 received a reduced dose of $\mathrm{TMZ}$ due to pancytopenia with eventual discontinuation after 9 cycles due to several delays and dose reductions of TMZ. All three patients demonstrated clinical stability, decrease in tumor size radiologically and decreasing SPRL. Previous studies have published favorable responses of corticotroph tumors with TMZ treatment, however, the optimal dose, and the number of cycles is unclear for these other subtypes as well. Long-term TMZ treatment could be tolerated and may be necessary for some refractory prolactinomas, as Patient 1 recurred on surveillance after 18 monthly cycles of TMZ, and again had a good response to $T M Z$ re-challenge, hence making this is a unique case as previously published case series have shown that response to retreatment of refractory pituitary tumors with $\mathrm{TMZ}$ is poor. [8]. In addition, for patients with refractory prolactinomas with concurrent paranoid schizophrenia who cannot receive DA, TMZ may be considered earlier in the treatment course. The tumor tissue was not tested for MGMT status in our patient cohort and therefore is a limitation of this study. Larger prospective studies are needed to 
ascertain whether earlier treatment with TMZ may spare standard treatment modalities, such as radiation.
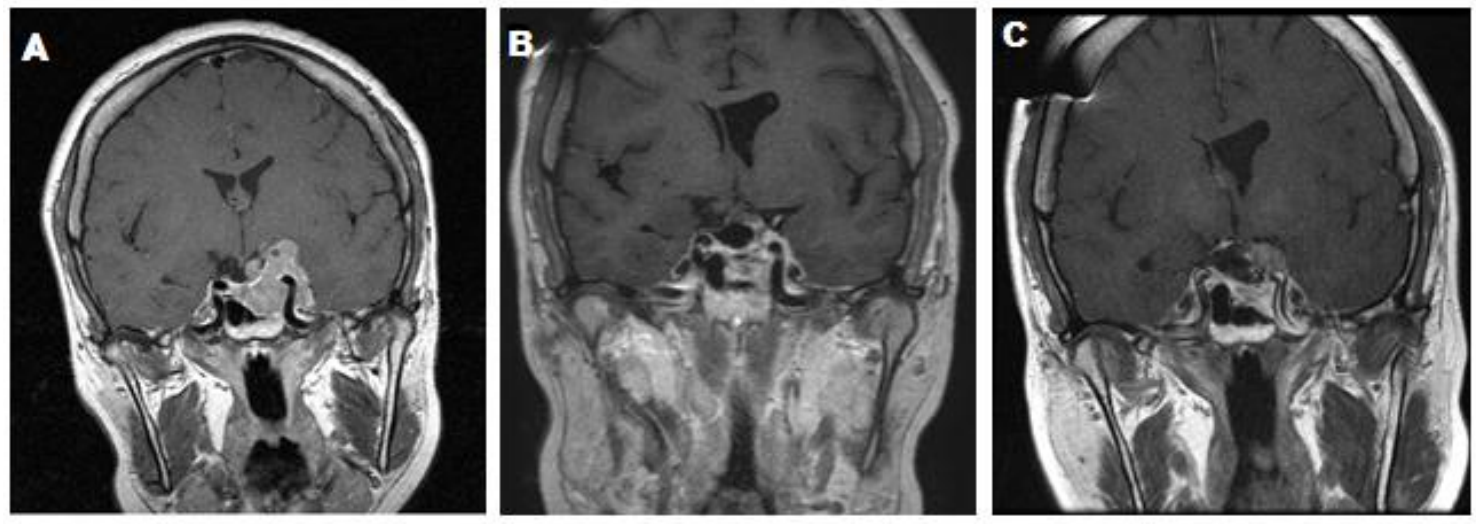

Figure 1 Patient 1 Images A to C: Contrast-enhanced coronal brain MRIs. A: 10 years after initial diagnosis. The patient failed prior DA therapy, surgery and radiosurgery. B: Decrease in tumor size 6 months into TMZ therapy. C: Extension of left suprasellar enhancement during TMZ holiday.

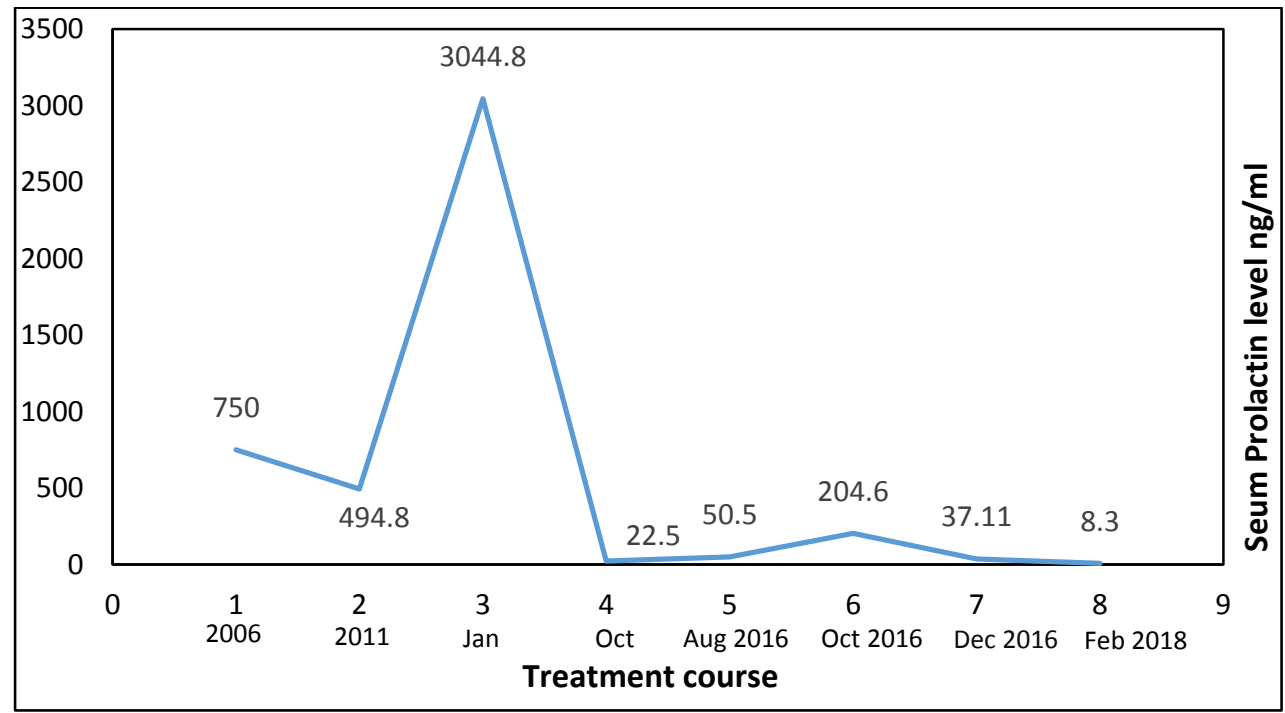

Figure 2 Graph showing SPRL for patient 1 over time since diagnosis. 1. SPRL after DA treatment; 2. Failed surgery and Cyber Knife; 3. SPRL prior to starting TMZ; 4. Completion of 18 cycles of TMZ; 5. SPRL on surveillance; 6 . Radiographic recurrence; 7. After 2 cycles of TMZ retreatment; 8. Completion of 21 cycles of TMZ. Note: SPRL from 1996 is unavailable. Normal SPRL in non-pregnant females: $5-40 \mathrm{ng} / \mathrm{ml}$ or $106-850 \mathrm{mIU} / \mathrm{L}$.

\section{Acknowledgements}

This research is supported partially by grants from AbbVie Inc. and Leadiant Biosciences. The authors thank Samarpita Sengupta, Ph.D. and Elise Burger for their help with the manuscript. 


\section{Author Contributions}

All authors contributed equally to the conception or design of the work, data collection, data analysis and interpretation, drafting the article, critical revision of the article and final approval of the version to be published.

\section{References}

1. Moisi M, Cruz AS, Benkers T, Rostad S, Broyles FB, Yuen K, et al. Treatment of Aggressive Prolactin-Secreting Pituitary Adenomas with Adjuvant Temozolomide Chemotherapy: A Review. Cureus. 2016; 8: e658.

2. Glezer A, Bronstein MD. Prolactinomas. Endocrinol Metab Clin North Am. 2015; 44: 71-78.

3. Cohen-Inbar O, Xu Z, Schlesinger D, Vance ML, Sheehan JP. Gamma Knife radiosurgery for medically and surgically refractory prolactinomas: long-term results. Pituitary. 2015; 18: 820-830.

4. Fadul CE, Kominsky AL, Meyer LP, Kingman LS, Kinlaw WB, Rhodes CH, et al. Long-term response of pituitary carcinoma to temozolomide. Report of two cases. J Neurosurg. 2006; 105: 621-626.

5. Syro LV, Uribe H, Penagos LC, Ortiz LD, Fadul CE, Horvath E, et al. Antitumour effects of temozolomide in a man with a large, invasive prolactin-producing pituitary neoplasm. Clin Endocrinol (Oxf). 2006; 65: 552-553.

6. Halevy C, Whitelaw BC. How effective is temozolomide for treating pituitary tumours and when should it be used? Pituitary. 2017; 20: 261-266.

7. Bengtsson D, Schroder HD, Andersen M, Maiter D, Berinder K, Feldt Rasmussen U, et al. Longterm outcome and MGMT as a predictive marker in 24 patients with atypical pituitary adenomas and pituitary carcinomas given treatment with temozolomide. J Clin Endocrinol Metab. 2015; 100: 1689-1698.

8. McCormack Al, Dekkers O, Petersenn S, Popovic V, Trouillas J, Raverot G, et al. Treatment of aggressive pituitary tumours and carcinomas: results of a European Society of Endocrinology (ESE) survey 2016. Eur J Endocrinol. 2018; EJE-17-0933.

9. Stupp R, Mason WP, Van Den Bent MJ, Weller M, Fisher B, Taphoorn MJ, et al. Radiotherapy plus concomitant and adjuvant temozolomide for glioblastoma. New Engl J Med. 2005; 352: 987-996.

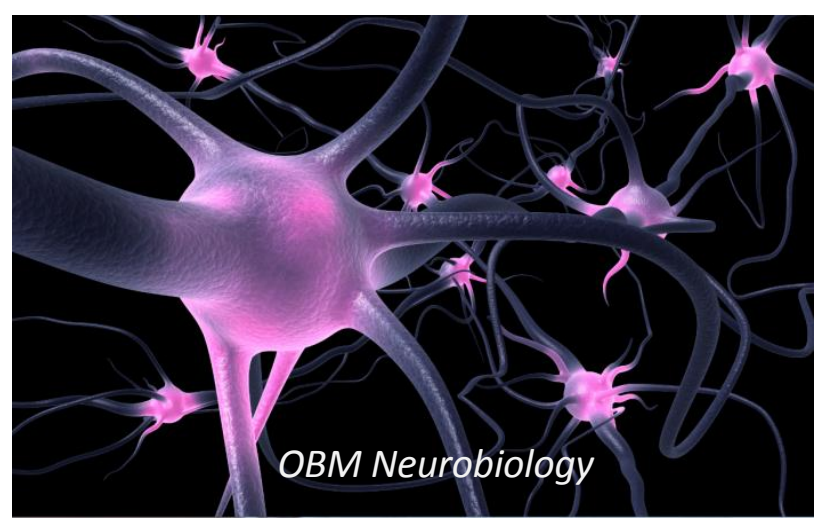

Enjoy OBM Neurobiology by:

1. Submitting a manuscript

2. Joining volunteer reviewer bank

3. Joining Editorial Board

4. Guest editing a special issue

For more details, please visit: http://www.lidsen.com/journals/neurobiology 